\title{
Coherent carrier dynamics in semiconductor superlattices
}

\author{
Enrique Diez, ${ }^{a}$ Rafael Gómez-Alcalá, ${ }^{b}$ Francisco Domínguez-Adame, ${ }^{c}$ Angel Sánchez,${ }^{a}$ and \\ Gennady P. Berman ${ }^{d}$ \\ ${ }^{a}$ GISC, Departamento de Matemáticas, Universidad Carlos III, E-28911 Leganés, Madrid, Spain \\ ${ }^{b}$ Departamento de Tecnologías de las Comunicaciones, Universidade de Vigo, E-36200 Vigo, \\ Spain \\ ${ }^{c}$ GISC, Departamento de Física de Materiales, Universidad Complutense, E-28040 Madrid, Spain \\ ${ }^{d}$ Theoretical Division and CNLS, B213, Los Alamos National Laboratory, Los Alamos, New \\ Mexico 8754 and Kirensky Institute of Physics, 660036 Krasnoyarsk, Russia
}

(January 6, 2014)

\begin{abstract}
We investigate the coherent dynamics of carriers in semiconductor superlattices driven by ac-dc electric fields. We solve numerically the timedependent effective-mass equation for the envelope function. We find that carriers undergo Rabi oscillations when the driving frequency is close to the separation between minibands.
\end{abstract}

Typeset using REVTEX 
Recent advances in laser technology make possible to drive semiconductor nanostructures with intense coherent ac-dc fields. This opens new research fields in time-dependent transport in mesoscopic systems [1,2] and puts forward the basis for a new generation of ultra-high speed devices. Artificial two-level semiconductor nanostructures acting as switching devices based on Rabi oscillations (RO's) have been already suggested [3]. Moreover, resonant phonon-assisted tunneling through a double quantum dot could be used as an efficient electron pump from spatial RO's [⿴囗⿰丿㇄𠃊].

Zhao et al. have investigated analytically a tight-binding model of a two-band system in a time-dependent ac-dc field in the weak coupling limit [5]. They identified RO's between Bloch bands under resonant conditions, which reveal the existence of quasienergy bands and fractional Wannier-Stark ladders. The advances achieved in molecular beam epitaxy, which allow to fabricate semiconductor superlattices (SL's) tailored with the desired conductionband profiles, make these systems ideal candidates to propose experiments on coherent carrier dynamics. However, the tight-binding approximation presents some limitations to describe actual semiconductor superlattices (SL's) when the coupling between neighboring quantum wells is not weak. Thus, in order to experimentally access the validity of theoretical predictions, one should use a more realistic model. In this Letter we present an effectivemass model beyond the tight-binding approximation, containing all ingredients of actual SL's, namely finite interband coupling and multiband scattering.

We consider electron states close to the conduction-band edge and use the effective-mass approximation. The electron wave packet satisfies the following equation

$$
\begin{aligned}
& i \hbar \frac{\partial \Psi(x, t)}{\partial t}= \\
& {\left[-\frac{\hbar^{2}}{2 m^{*}} \frac{d^{2}}{d x^{2}}+V_{\mathrm{SL}}(x)-e F x \sin \left(\omega_{\mathrm{ac}} t\right)\right] \Psi(x, t),}
\end{aligned}
$$

where $x$ is the coordinate in the growth direction, $F$ and $\omega$ are the strength and the frequency of the ac field. The SL potential at flat band is $V_{\mathrm{SL}}(x)=\Delta E_{c}$ if $x$ lies inside the barriers and zero otherwise, $\Delta E_{c}$ being the conduction-band offset. We have considered a constant effective-mass $m^{*}$ for simplicity. 
The band structure at flat band is computed by using a finite-element method [6]. The eigenstate $j$ of the band $i$ with eigenenergy $E_{i}^{(j)}$ is denoted as $\psi_{i}^{(j)}(x)$. A good choice for the initial wave packet is provided by using a linear combination of the eigenstates belonging to the first miniband. For the sake of clarity we have selected as the initial wave packet $\Psi(x, 0)=\psi_{i}^{(j)}(x)$, although we have checked that this assumption can be dropped without changing our conclusions. The subsequent time evolution of the wave packet $\Psi(x, t)$ is calculated numerically by means of an implicit integration schema [7]. In addition to $\Psi(x, t)$ we also compute the probability of finding an electron, initially in the state $\Psi(x, 0)=\psi_{i}^{(j)}(x)$, in the state $\psi_{k}^{(j)}(x)$

$$
P_{i k}^{(j)}(t)=\int_{-\infty}^{\infty} d x \Psi^{*}(x, t) \psi_{k}^{(j)}(x)
$$

We present here the results for a SL with 10 periods of $100 \AA$ GaAs and $50 \AA \mathrm{Ga}_{0.7} \mathrm{Al}_{0.3} \mathrm{As}$ with band offset $\Delta E_{c}=250 \mathrm{meV}$ and $m^{*}=0.067 \mathrm{~m}, m$ being the free electron mass. We consider electric field $F=25 \mathrm{kV} / \mathrm{cm}$ as a typical value, although similar results are observed for other strengths. Figure 1 (a) displays $P_{01}^{(5)}(t)$ for $F=25 \mathrm{kV} / \mathrm{cm}$ at the resonant frequency $\omega_{\mathrm{ac}}=\left(E_{1}^{(5)}-E_{0}^{(5)}\right) / \hbar=150 \mathrm{THz}$. Thus, we are monitoring the transitions between the central state $(j=5)$ in the first miniband to the central state in the second miniband as a function of time. We observe the occurrence of very well defined RO's with an amplitude close to 0.3. Summing up the probabilities of the rest of states in the second miniband, the probability of finding the electron in this band is very close to unity $(\sim 0.99)$. The frequency of the RO's, obtained performing the fast Fourier transform $(\mathrm{FFT})$ of $P_{01}^{(5)}(t)$, is $\omega_{\text {Rabi }}=19.18 \mathrm{THz}$. The probability $P_{01}^{(5)}(t)$ is dramatically reduced when the ac driving field is out of resonance, as shown in Fig. 1(b) for $\omega_{\mathrm{ac}}=100 \mathrm{THz}$. The FFT of those date reveals no specific features besides the peak at the driving frequency $\omega_{\mathrm{ac}}$.

In a pure two-level system, a straightforward perturbation calculation yields $\omega_{\text {Rabi }}=$ $\left|F_{01}\right| / \hbar$ at resonance, where $F_{01}$ is the matrix element of the perturbation between the ground state and the first excited state. Thus, $\omega_{\text {Rabi }}$ is linear in the electric field in a pure two-level system. Although the SL is not a pure two-level system, we realize that this linear 
dependence still holds, as we can see in Fig. 2.

As an estimation of the leakage current that one could observe in electron pumping devices based in RO's in SL's, we have studied the integrated density in the right part of the SL, defined as

$$
P_{T}(t)=\int_{x_{r}}^{\infty}|\Psi(x, t)|^{2} d x
$$

where $x_{r}$ is the coordinate of the right edge of the SL. Figure 3 shows the results at the resonant frequency $\omega_{\mathrm{ac}}=150 \mathrm{THz}$ as well as out of resonance when $\omega_{\mathrm{ac}}=200 \mathrm{THz}$. Under resonant conditions the wave function is emitted by bursts from the SL region every time a RO has been completed. On the contrary, the tunneling across the whole SL is negligible when the ac driving frequency is not very close to the resonant one.

In summary, we have studied the coherent carrier dynamic in semiconductor SL's driven by an intense ac-dc field. We found that the electron can perform RO's under resonant conditions as in pure two-level systems. The Rabi frequency of the oscillations depends linearly on the strength of the electric field. We have shown that electrons are emitted by bursts under resonant conditions, whereas the tunneling probability in vanishingly small out of resonance. Therefore, we suggest that semiconductor SL's driven by an intense acdc field may be used as an efficient electron pumping device in $\mathrm{THz}$ science. Finally, we should mention that we have not considered the role of imperfections or any other scattering mechanism that could result in a reduction of the carrier coherence. Our preliminary results show that scattering by interface roughness does not strongly modify the above picture. Further work along these lines is currently in progress.

E. D. thanks to Antti-Pekka Jauho illuminating conversations and his warm hospitality at Mikroelektronik Centret where this paper was written in part. Work at Leganés and Madrid is supported by CICYT (Spain) under projects MAT95-0325 and DGES PB96-0119. E. D. gratefully acknowledges partial support from Fundación Universidad Carlos III de Madrid. G. P. B. gratefully acknowledges partial support by the Defense Advanced Research Projects Agency. 


\section{REFERENCES}

[1] J. B. Pieper and J. C. Price, Phys. Rev. Lett. 72, 3586 (1994).

[2] S. T. Cundiff, A. Knorr, J. Feldmann, S. W. Koch, E. O. Göbel and H. Nickel, Phys. Rev. Lett. 73, 1178 (1994).

[3] T. Martin and G. P. Berman, Phys. Lett. A 196, 65 (1994).

[4] C. A. Sttaford and N. S. Wingreen, Phys. Rev. Lett. 76, 1916 (1996).

[5] X. -G. Zhao, G. A. Georgakis, and Q. Niu, Phys. Rev. B 54, R5235 (1996).

[6] K. Nakamura, A. Shimizu, M. Koshiba, K. Hayata, IEEE J. Quantum Electron. 25, 889 (1989).

[7] E. Diez, A. Sánchez, and F. Domínguez-Adame, Phys. Rev. B 54, 14550 (1996). 


\section{FIGURES}

FIG. 1. The probability of finding an electron, initially situated in the state $\psi_{0}^{(5)}$, in the state $\psi_{1}^{(5)}$ as a function of time for $F=25 \mathrm{kV} / \mathrm{cm}$, when the ac field (a) is tuned to the resonant frequency $\omega_{\mathrm{ac}}=150 \mathrm{THz}$ and $(\mathrm{b})$ is out of resonance $\omega_{\mathrm{ac}}=100 \mathrm{THz}$. Note the different vertical scales.

FIG. 2. Rabi frequency as a function of the dc field when the driving frequency is tuned to the resonant frequency $\omega_{\mathrm{ac}}=150 \mathrm{THz}$

FIG. 3. $P_{T}(t)$ as function of time at at the resonant frequency $\omega_{\mathrm{ac}}=150 \mathrm{THz}$ (solid line) and out of resonance $\omega_{\mathrm{ac}}=200 \mathrm{THz}$ (dashed line). 


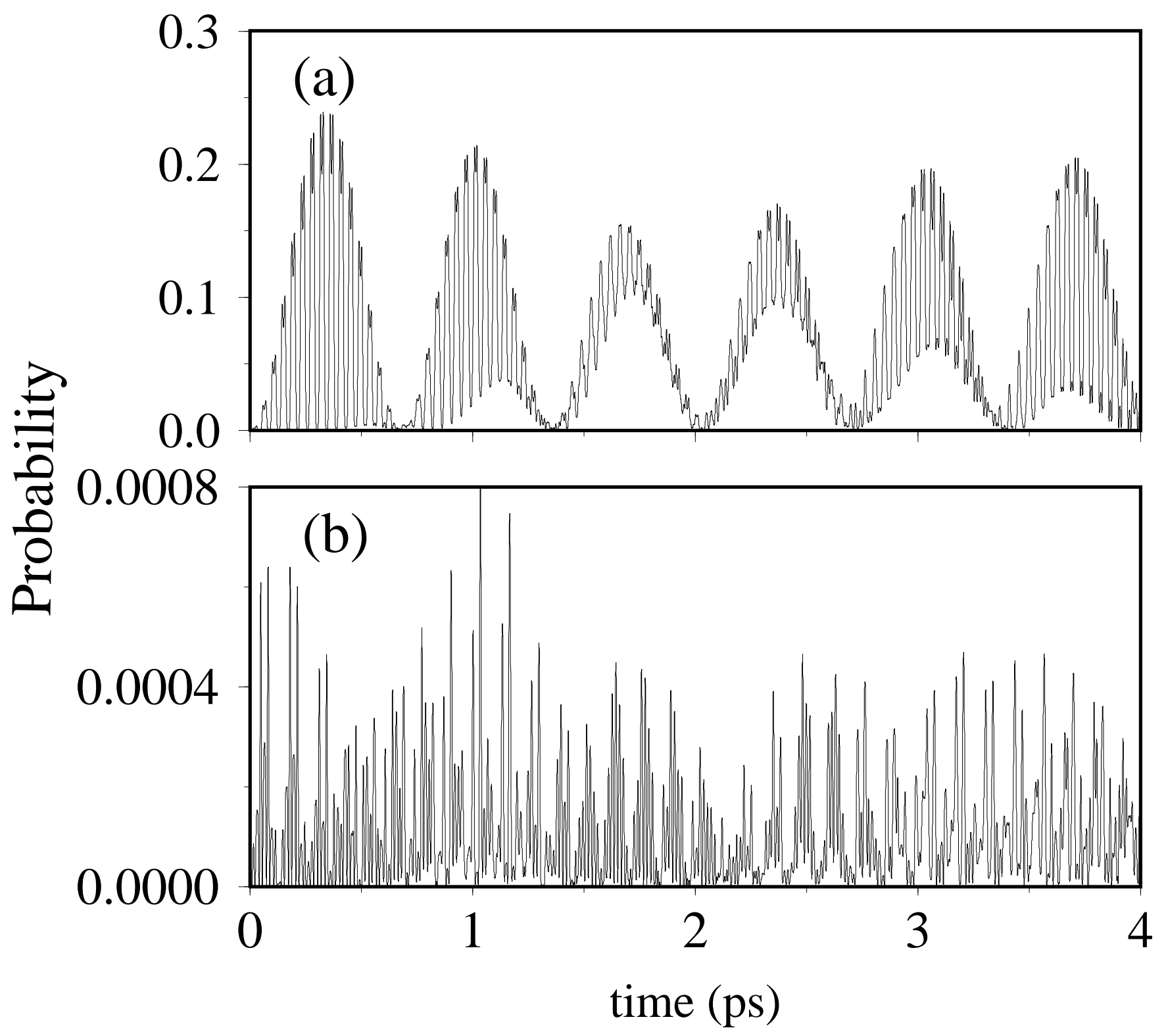




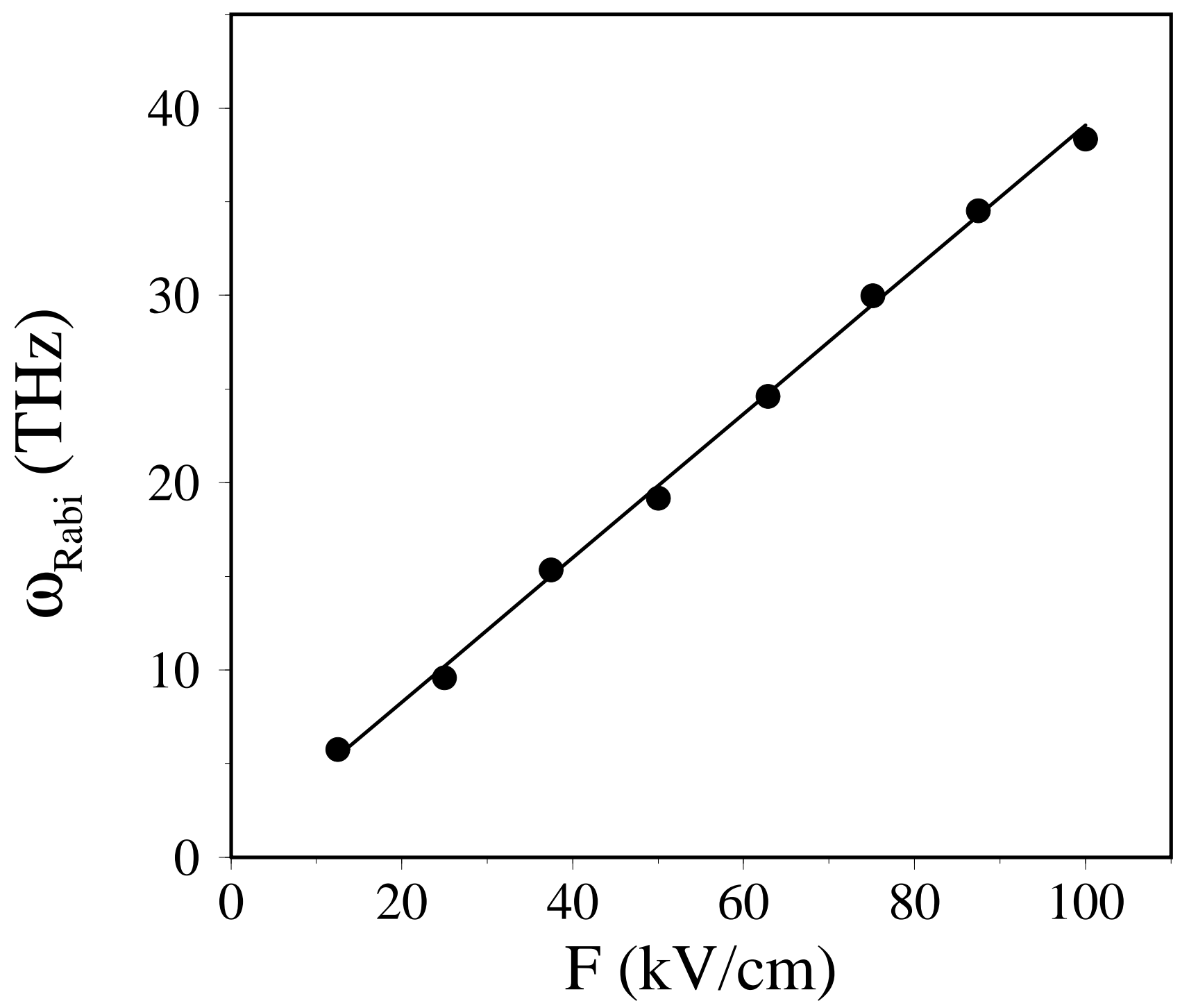




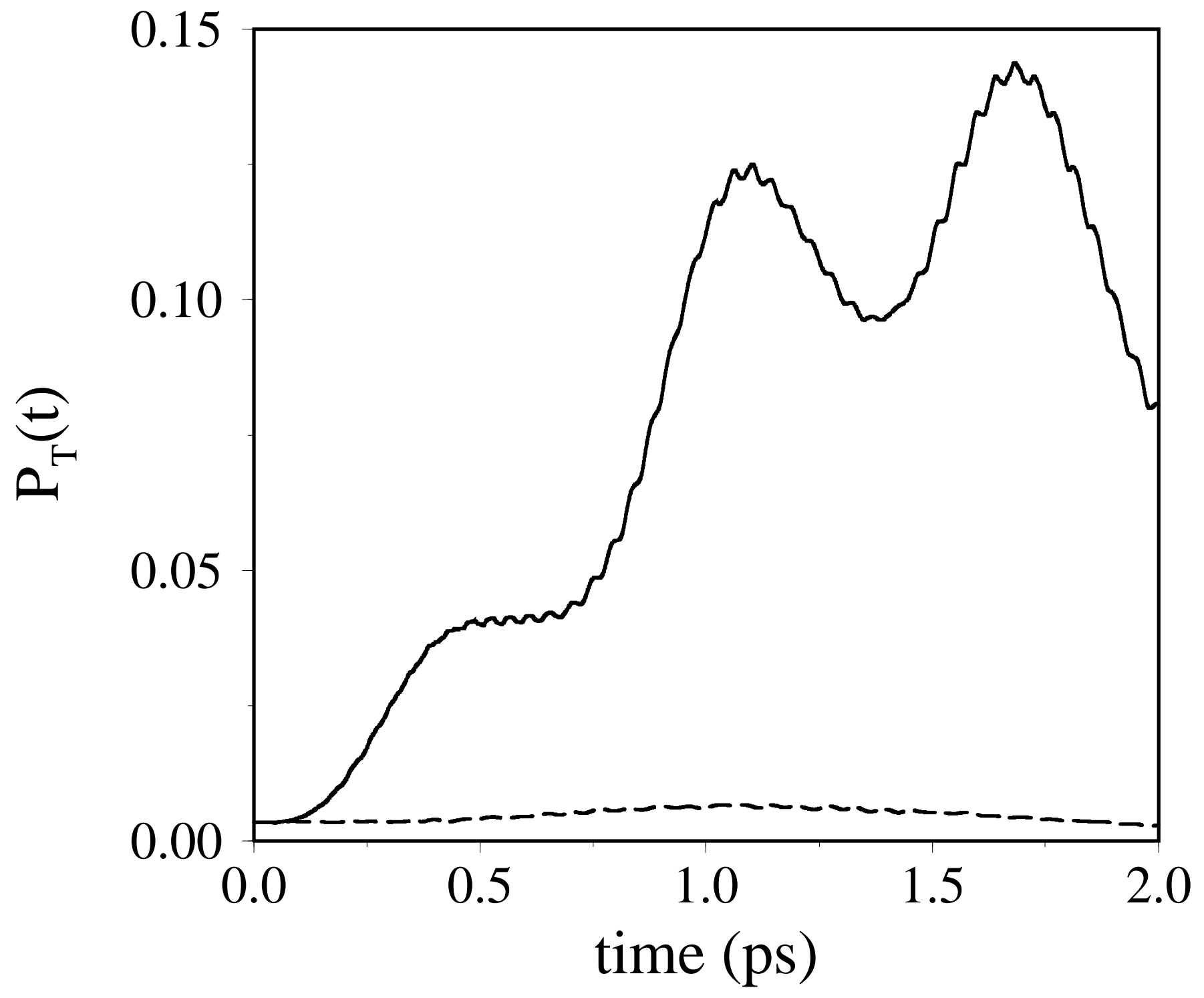

